\title{
Detection of incomplete enclosures of rectangular shape in remotely sensed images
}

\author{
Igor Zingman, Dietmar Saupe \\ Computer and Information Science, University of Konstanz \\ Germany \\ first. lastname@uni-konstanz.de
}

\author{
Karsten Lambers \\ IADK, University of Bamberg \\ Germany \\ first.lastname@uni-bamberg.de
}

\begin{abstract}
We develop an approach for detection of ruins of livestock enclosures in alpine areas captured by high-resolution remotely sensed images. These structures are usually of approximately rectangular shape and appear in images as faint fragmented contours in complex background. We address this problem by introducing a new rectangularity feature that quantifies the degree of alignment of an optimal subset of extracted linear segments with a contour of rectangular shape. The rectangularity feature has high values not only for perfect enclosures, but also for broken ones with distorted angles, fragmented walls, or even a completely missing wall. However, it has zero value for spurious structures with less than three sides of a perceivable rectangle. Performance analysis using large imagery of an alpine environment is provided. We show how the detection performance can be improved by learning from only a few representative examples and a large number of negatives.
\end{abstract}

\section{Introduction}

We address the problem of detecting remains of manmade enclosures used to hold livestock in grassland of mountainous regions. The livestock enclosures (LE) are of special archaeological interest because they offer important insights into historical development of alpine pastoralism. Their automated spotting was questioned in a recent archaeological project [18]. Examples of such enclosures are shown in Fig. 1. These structures are usually composed of linear walls that may be heavily ruined. The most common shape of LE resembles a rectangular contour with greatly varying size and aspect ratio. Rectangle angles may deviate from right angles, and rectangle sides may be fragmented. The angle between adjacent fragments of the same (broken) side may deviate from 180 degrees. Moreover, the rectangular contours are sometimes incomplete such that even an entire side may be missing.
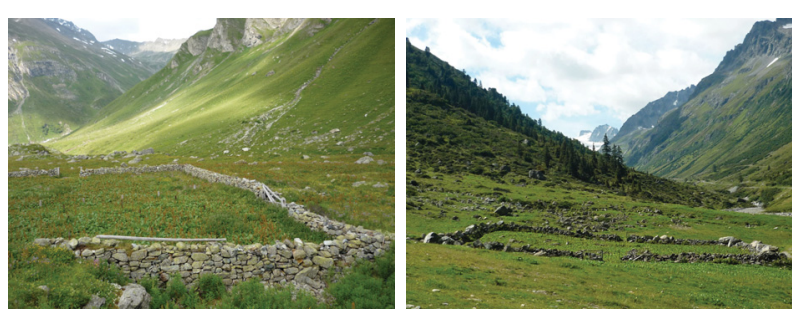

Figure 1. Livestock enclosures (LE) in alpine environment

We use satellite and aerial images of $0.5 \mathrm{~m}$ resolution where the width of linear walls does not exceed two pixels. The ruined walls are of low height, which results in low contrast linear features in the images. The spectral properties of LE are similar to the spectral properties of the surrounding terrain, rocks, and other irrelevant objects. The first row of Fig. 5 shows a satellite and an aerial image with structures corresponding to the LE shown in Fig. 1. Nearby irrelevant structures, such as rivers, trails, or rocks, are often of similar or higher contrast either due to larger size (e.g. big rocks) or distinctive spectral properties (e.g. rivers). Detection of such faint structures in a complex terrain is a challenging task. Even the detection of easily modeled circular soil structures [32] had very limited success due to their low contrast and complex terrain. Only few examples of LE are available in our case, which presents another difficulty making most approaches that learn from the data inappropriate. Because of these difficulties, commonly used methods for rectangle detection are hardly applicable.

In contrast to spectral properties, the geometrical properties of LEs appear to be more distinctive and do not depend on image modality and conditions under which an image was captured. We therefore develop a measure that quantifies the distinctive geometry of approximately rectangular enclosures. Our approach relies on a new rectangularity feature that discriminates rectangular patterns from other structures in complex cluttered background. The feature is based on a prior model of a fragmented rectangle, which is a convex polygon with constrained angles. 


\subsection{Related work}

Detection of rectangular structures has previously been addressed in different contexts. Examples are detection of buildings in remotely sensed images $[24,19,14,3,12,15$, $1,31,30,23,26]$, traffic signs [21, 13, 22], and particles of a rectangular shape in cryo-electron microscopy images $[36,35]$. The methods used were based on Markov Random Fields [15, 21], Marked Point Processes [1, 26], search on a graph $[14,38]$, Hough Transform and other voting schemes $[3,12,36,13,22]$, template matching [25], aggregation of local features [1, 23, 31], and heuristic rules [19].

Most techniques for detection of rectangular structures dealt with buildings in remotely sensed images. For example, in the graph-based approach [14], a search for cycles was used to generate building hypotheses. The search was accompanied by an extensive set of rules and thresholds, which limits the robustness of the approach.

Markov Random Fields (MRF) were used in [15] to delineate buildings. More recently, a similar approach was used in [21] for detection of traffic signs in color images. The approach is sensitive to inaccuracy of extracted edges and cannot detect incomplete rectangles, as it requires the presence of all four sides of a rectangular structure. The marked point processes (MPP) [4] recently became popular for extraction of various structures in remotely sensed images, including buildings (e.g. in [1, 26]). The MPP proved to be very powerful when applied to real data. However, these stochastic methods are still computationally expensive. Similarly to the MRF, they may not converge to a globally optimal solution and usually need careful tuning of a large number of parameters. Attempts have recently been made to address some of these problems, which are crucial for the analysis of large images. In [33] substantial improvements in performance have been achieved for the extraction of line networks (roads and rivers). In this work also the potential of GPUs was efficiently exploited.

An approach for detection of rectangular contours based on the Hough transform was developed in [12]. The approach relies on certain strict geometrical rules making it not suitable for detection of fragmented or incomplete structures. It may also result in detection of rectilinear configurations that cannot form a rectangular contour. Detection of such configurations is prevented in our approach by adding a convexity constraint.

In [31] a set of local features that carried local corner information were used to produce a probability map of building rooftops. Unfortunately, in the case of fragmented enclosures corners are not reliable features. Moreover, local features in general do not suffice in the case of faint contours appearing in a cluttered background. A more global description that takes into account spatial relations between local features is necessary. For example, in $[1,23]$ the gradient orientation density function (GODF) was computed from image gradients. A correlation of this function with a mixture of two Gaussians having mean values separated by ninety degrees served as a GODF-based feature indicating the presence of buildings.

Although there is a variety of methods developed for building detection, they are not applicable to our task because buildings are much more salient structures. In contrast to building rooftops, walls of ruined livestock enclosures are narrow and are of low hight (low contrast features), may be highly fragmented, or even completely missing. Higher contrast irrelevant structures may appear inside or outside of rectangular structures in the immediate neighborhood. Various cues (rooftop color, shadows, 3D cues etc.) usually employed in building detection algorithms are not available.

\subsection{Overview of our approach}

Our approach relies on the basic detection scheme, which includes localization of candidate points, we presented in [38]. A binary map of edges accompanied by angle information is computed first. Linear segments are then found and modeled by a few parameters with the use of a local Hough transform. An undirected graph is constructed, nodes of which correspond to linear segments and graph edges encode spatial relations between linear segments. Particularly, we use angle and convexity properties to encode spatial relations. Due to the construction of the graph, its maximal cliques correspond to valid configurations of linear segments. The valid configurations are then ranked by a new rectangularity measure that encodes the goodness of grouping the segments into a rectangular structure (Sec. 2.3). In contrast to [38], the new rectangularity measure does not rely on a heuristic partitioning of the set of linear segments into four subsets. Hard decisions are softened. Configurations better matching the rules result in a higher rectangularity measure. The rectangularity feature is defined as the maximal rectangularity measure of all valid configurations (Sec. 2.4). In practice, the number of corresponding maximal cliques within the analysis window is low, allowing exact and efficient maximization. The resulting rectangularity feature captures the presence of $\Pi$-like structures and is robust to their fragmentation.

We introduce the new rectangularity feature in Sec. 2. In Sec. 2.5 we show how to improve the detection performance based solely on the rectangularity feature by introducing an additional feature proportional to enclosure size and learning from the large number of negative examples and just few positives. Using remotely sensed imagery in Sec. 3, we, for the first time, evaluate the performance of the normalized maximal rectangularity (NMR) measure [38] and the introduced here rectangularity features for enclosure detection. We also compare them with the GODF-based feature from [1] used for building detection. We conclude in Sec. 4 and 
discuss future directions.

\section{Measuring structure rectangularity}

We introduce a rectangularity feature $f_{R}$ computed from a set of linear segments $\mathbf{W}=\left\{\mathcal{S}_{i}, i=1, \ldots, m\right\}$ that were extracted from a gray-scale image.

\subsection{Grouping edge points into linear segments}

In Sec. 3.1 we provide details on approaches we used to extract ridges and valleys (bar edges) and to detect candidate locations. Given a candidate location and edge points accompanied by estimated orientations we extract and parameterize linear segments, each of which is a group of aligned edge points. Linear segments are represented by a triple of parameters $(\theta, r, l)$ found by the use of a local Hough transform centered at the candidate points. We use the Hough transform in the form introduced in [6], where a line is defined by the orientation $\theta$ of the normal and a distance $r$ from the origin

$$
r=x \cos \theta+y \sin \theta .
$$

The spatial coordinates of an edge point are $x, y, \theta \in$ $[0,360)$, and $r \in(0, \infty)$. A peak at $(\theta, r)$ in the Hough plane corresponds to a line. The peaks are detected as regional maxima in the Hough plane that was discretized with $\Delta \theta=3^{\circ}$ and $\Delta r=1$ pixel. The detected line corresponds to either a single connected linear segment $\mathcal{S}$, or to several aligned connected components. In the latter case, the connected components with gaps smaller than a predefined threshold ( 3 pixels in our experiments) are considered a single linear segment (see the segment $\mathcal{S}_{j}$ in Fig. 2), otherwise they are considered separate linear segments. This was not allowed in [38], where Hough lines always corresponded to a single linear segment (connected or fragmented), which restricted the number of candidate configurations of linear segments. The parameter $l$ in the triple $(\theta, r, l)$ is the number of points that belong to the linear segment. To better relate the parameter $l$ to the length and avoid its dependence on the width of the extracted edges, we perform their thinning [17] prior to clustering in a Hough plane.

Since edges were extracted together with their orientations, $r$ can be directly computed for each edge point $(x, y)$ using Eq. (1). Thus, each edge point votes for a single point in the $(\theta, r)$ plane instead of voting for a curve as suggested in [6]. This idea, which was used already in [7] for clustering of short ridge features, considerably eases extraction of meaningful peaks in the Hough plane.

\subsection{Valid configurations of linear segments}

Below we define a valid configuration of linear segments $\mathbf{C} \subseteq \mathbf{W}$ that can be a part of a rectangular structure. We require angles $\beta_{k, j}$ between linear segments $\mathcal{S}_{k}, \mathcal{S}_{j} \in \mathbf{C}$ of the valid configuration to be close to either zero, $180^{\circ}$, or right angles. An angle tolerance $\alpha$ will be set to control the strictness of the angle constraint. We define $\beta_{k, j}$ as

$$
\beta_{k, j}=\min \left(\left|\theta_{\mathcal{S}_{k}}-\theta_{\mathcal{S}_{j}}\right|, 360-\left|\theta_{\mathcal{S}_{k}}-\theta_{\mathcal{S}_{j}}\right|\right) .
$$

Note that $\beta_{j, k}=\beta_{k, j}$ and $\beta \in[0,180]$, since $\theta \in[0,360)$. The angle constraint alone does not suffice to restrict configurations to be perceptually close to rectangles or rectangle parts. We therefore define a second constraint that requires the valid configuration to be nearly convex in the sense that extension of all linear segments of the configuration can form an nearly convex contour. The convexity tolerance $t$ will be defined to control the strictness of the convexity constraint. For a convex configuration of linear segments it is required that a half plane generated by each segment includes all other segments of the configuration. Additionally, we require that all these half planes contain the candidate point around which we search for a rectangular structure. Pair-wise convexity constraints suffice to verify the convexity of a configuration containing the given candidate point. We define the pair-wise convexity measure $\tau$ for a pair of linear segments $\mathcal{S}_{k}, \mathcal{S}_{j}$, each with corresponding attributes of size $l_{\mathcal{S}}$, orientation $\theta_{\mathcal{S}}$, and distance $r_{\mathcal{S}}$ to the candidate point $p_{0}$, as

$$
\begin{aligned}
\tau_{k, j} & =\max \left(\tilde{\tau}_{k, j}, \tilde{\tau}_{j, k}\right), \\
\tilde{\tau}_{k, j} & =\frac{1}{l_{j}} \sum_{p \in \mathcal{S}_{j}} H\left(\left(p-p_{0}\right)^{T} \cdot n_{k}-r_{k}\right),
\end{aligned}
$$

where $n_{k}=\left(\cos \theta_{k}, \sin \theta_{k}\right)^{T}$ is the unit normal of $\mathcal{S}_{k}$ and $H(u)$ is an indicator function equal one for $u>0$ and zero otherwise. $\tilde{\tau}_{k, j}$ measures the relative number of points in the segment $\mathcal{S}_{j}$ that are behind the segment $\mathcal{S}_{k}$, relative to the given candidate point $p_{0}$ as illustrated in Fig. 2. Note that $\tau \in[0,1]$, and $\tau_{k, j}=\tau_{j, k}$, while $\tilde{\tau}_{k, j} \neq \tilde{\tau}_{j, k}$.

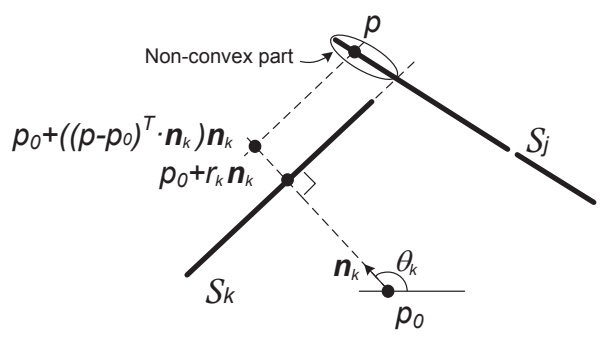

Figure 2. The fraction of points $p$ of $\mathcal{S}_{j}$ that violates the convexity constraint relative to $\mathcal{S}_{k}$ and $p_{0}$ is given by $\tilde{\tau}_{k, j}$. Note that linear segments can be fragmented having small gaps as in $\mathcal{S}_{j}$.

Definition 1. Let $\alpha \in[0,45], t \in[0,1]$, a candidate point $p_{0}$, and a configuration $\mathbf{C}$ of linear segments be given. If for all pairs $\mathcal{S}_{k}, \mathcal{S}_{j} \in \mathbf{C}, j \neq k$, one of the inequalities of the angle constraint

$$
\beta_{k, j} \leq \alpha \text { or }\left|90-\beta_{k, j}\right| \leq \alpha \text { or } 180-\beta_{k, j} \leq \alpha
$$


and the convexity constraint

$$
\tau_{k, j} \leq t
$$

both hold, then $\mathbf{C}$ is called a $(\mathrm{t}, \alpha)$-valid configuration located around $p_{0}$, and denoted by $\mathbf{C}_{p_{0}}^{t, \alpha}$.

For the sake of brevity, we usually omit the indices $t$, $\alpha$ and the reference point $p_{0}$, mentioning that $\mathbf{C}$ is a valid configuration. Valid configurations include not only perfect rectangles, but also convex polygons or their parts with angles around either 90 or 180 degrees. This is important in practice since approximately rectangular structures are better modeled by such polygons rather than by perfect rectangles.

\subsection{Rectangularity measure of a valid configuration}

A couple of poorly aligned short segments can be a valid configuration as far as the tolerances $t, \alpha$ allow. There is a need to rank valid configurations according to their similarity to a canonical rectangle. To find and rank valid configurations we construct an undirected graph $\mathbf{G}^{w}$ from the given set $\mathbf{W}$ of linear segments in a window centered at a candidate point $p_{0}$. The graph $\mathbf{G}^{w}$ has nodes $j=1, . ., m$ corresponding to the segments $\mathcal{S}_{1}, . ., \mathcal{S}_{m} \in \mathbf{W}$. Each node $j$ is attributed by a triple of parameters $\left(\theta_{j}, r_{j}, l_{j}\right)$, i.e. orientation, distance to the reference point $p_{0}$, and size of the linear segment. An edge $\{k, j\}$ is attributed with the angle $\beta_{k, j}$ and the pair-wise convexity $\tau_{k, j}$ of the corresponding pair of segments $\mathcal{S}_{k}, \mathcal{S}_{j}$. An edge $\{k, j\}$ is included in the graph $\mathbf{G}^{w}$ if $\beta_{k, j}$ and $\tau_{k, j}$ satisfy the constraints in Eqs. $(5,6)$. This attributed graph encodes properties of linear segments and their spatial relationships. Due to the graph construction and Definition 1, valid configurations $\mathbf{C}$ correspond to fully connected subgraphs $\mathbf{G}^{c}$, also called cliques, of the graph $\mathbf{G}^{w}$.

Below we introduce the new rectangularity measure $\rho\left(\mathbf{G}^{c}\right)$ that ranks a clique $\mathbf{G}^{\mathbf{c}}$ corresponding to a valid configuration $\mathbf{C} \subseteq \mathbf{W}$. We define the measure with the following properties in mind. The rectangularity measure shall yield higher values for configurations with

1. higher degree of convexity given by lower values of the convexity measure $\tau$

2. higher degree of angle alignments given by angles $\beta$

3. longer linear segments given by larger $l$.

In addition, the proposed rectangularity measure shall

4. have the increasing property $\rho\left(\mathbf{G}_{1}^{c}\right) \leq \rho\left(\mathbf{G}_{2}^{c}\right)$ for $\mathbf{G}_{1}^{c} \subseteq \mathbf{G}_{2}^{c}$. Thus, the rectangularity measure of a larger encompassing clique has a higher value

5. yield a zero value for configurations of linear segments with less than three sides of a rectangle.
We define the rectangularity measure of a graph clique $\mathbf{G}^{c}$ in terms of sums over its undirected edges $\{k, j\} \in E^{c}$

$$
\begin{aligned}
\rho\left(\mathbf{G}^{c}\right) & =\left(\left(\sum_{\{k, j\} \in E^{c}} l_{k} l_{j} \mathrm{f}_{90}\left(\beta_{k, j}\right) \mathrm{f}_{c v}\left(\tau_{k, j}\right)\right) \times\right. \\
& \left.\left(\sum_{\{k, j\} \in E^{c}} l_{k} l_{j} \mathrm{f}_{180}\left(\beta_{k, j}\right) \mathrm{f}_{c v}\left(\tau_{k, j}\right)\right)\right)^{\frac{1}{4}},
\end{aligned}
$$

where $\mathrm{f}_{90}, \mathrm{f}_{180}$, and $\mathrm{f}_{c v}$ are mode functions depicted in Fig. 3. $\mathrm{f}_{90}$ and $\mathrm{f}_{180}$ equal zero for angles $\beta$ that deviate from the mode center larger than the angle tolerance $\alpha$. $\mathrm{f}_{c v}$ equals zero for the convexity measure $\tau$ larger than the convexity tolerance $t$. In our experiments we used $\alpha=35^{\circ}$ and $t=$ 0.3 . The exact definition of the mode function is not critical and is not given here due to space constraints.
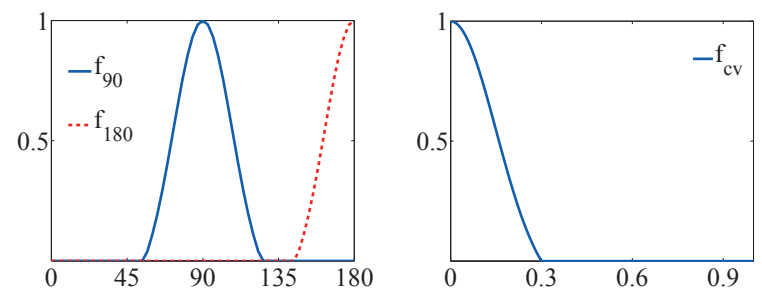

Figure 3. Functions $f_{90}$ (left figure, solid blue curve), $f_{180}$ (left figure, dashed red curve), and $\mathrm{f}_{c v}$ (right figure) used in the definition of the rectangularity measure in Eq. (7).

The first factor of $\rho\left(\mathbf{G}^{c}\right)$ in Eq. (7) yields a non-zero value only if the valid configuration $\mathbf{C}$ contains at least one pair of approximately perpendicular linear segments that fulfill the convexity constraint in Eq. (6). The second factor is non-zero only if the valid configuration contains at least one pair of approximately parallel linear segments ${ }^{1}$. The product of these two factors is non-zero only if the valid configuration $\mathbf{C}$ contains at least one pair of parallel and one pair of perpendicular linear segments. The angles between linear segments of these parallel and perpendicular pairs are restricted to be approximately 0,180 , or 90 degrees since $\mathbf{C}$ is a valid configuration with linear segments constrained by Eq. (5). Thus, a non-zero rectangularity measure insures a valid configuration $\mathbf{C}$ containing at least one triple of segments arranged in a $\Pi$-like structure, as stated in property 5 above. This property allows suppression of a large number of configurations originating from clutter (e.g. lines, corners, junctions etc.). It is easy to verify that the other four properties above are also satisfied by the rectangularity measure in Eq. (7).

\footnotetext{
${ }^{1} f_{c v}$ in the second term has only a small impact on results. It reduces the rectangularity measure for configurations with badly aligned opposite sides with a non-zero convexity measure.
} 
Note that the rectangularity measure is a function of graph node and edge attributes and does not require explicit partitioning of a valid configuration of linear segments into four subsets corresponding to four sides of a hypothesized rectangle as required in [38].

\subsection{Rectangularity feature}

Given a set of linear segments $\mathbf{W}$ in an analysis window, we define the rectangularity feature $f_{R}$ of the corresponding graph $\mathbf{G}^{w}$ using the rectangularity measure of its cliques $\mathbf{G}^{c}$. Let us denote the set of cliques as $\mathcal{K}\left(\mathbf{G}^{w}\right)$. The rectangularity feature of $\mathbf{G}^{w}$ is defined as

$$
f_{R}\left(\mathbf{G}^{w}\right)=\max _{\mathbf{G}^{c} \in \mathcal{K}\left(\mathbf{G}^{w}\right)} \rho\left(\mathbf{G}^{c}\right) .
$$

The corresponding optimal clique is

$$
\mathbf{G}_{\text {opt }}^{c}=\underset{\mathbf{G}^{c} \in \mathcal{K}\left(\mathbf{G}^{w}\right)}{\operatorname{argmax}} \rho\left(\mathbf{G}^{c}\right) .
$$

Due to the increasing property of $\rho$ (the fourth property of the rectangularity measure stated in Sec. 2.3), the maximum can be searched over the set of maximal cliques ${ }^{2}$ only, denoted here by $\mathcal{M}\left(\mathbf{G}^{w}\right)$

$$
f_{R}\left(\mathbf{G}^{w}\right)=\rho\left(\mathbf{G}_{o p t}^{c}\right)=\max _{\mathbf{G}^{c} \in \mathcal{M}\left(\mathbf{G}^{w}\right)} \rho\left(\mathbf{G}^{c}\right) .
$$

Since the set of maximal cliques $\mathcal{M}\left(\mathbf{G}^{w}\right) \subseteq \mathcal{K}\left(\mathbf{G}^{w}\right)$ is much smaller than the set of graph cliques $\mathcal{K}\left(\mathbf{G}^{w}\right)$, the number of times the rectangularity measure $\rho$ needs to be evaluated in Eq. (10) is considerably reduced in comparison to Eq. (8). Since, in addition, there are efficient algorithms for the search of maximal cliques [2], computing the rectangularity feature is not computationally demanding.

Fig. 4 (left) shows an example of a given set $W=$ $\left\{\mathcal{S}_{1}, \mathcal{S}_{2}, . ., \mathcal{S}_{6}\right\}$ of linear segments and the optimal configuration $\mathbf{C}_{\text {opt }}=\left\{\mathcal{S}_{1}, \mathcal{S}_{2}, \mathcal{S}_{3}, \mathcal{S}_{5}\right\}$ in red, while Fig. 4 (right) shows the corresponding graph $\mathbf{G}^{w}$ and the optimal maximal clique $\mathbf{G}_{\text {opt }}^{c}$ in red. There are two additional maximal cliques $\mathbf{G}_{1}^{c}$ and $\mathbf{G}_{2}^{c}$ and corresponding valid configurations $\mathcal{C}_{1}=\left\{\mathcal{S}_{2}, \mathcal{S}_{3}, \mathcal{S}_{4}, \mathcal{S}_{6}\right\}, \mathcal{C}_{2}=\left\{\mathcal{S}_{1}, \mathcal{S}_{2}, \mathcal{S}_{3}, \mathcal{S}_{4}\right\}$. They, however, have lower rectangularity values $\rho\left(\mathbf{G}_{1}^{c}\right)<$ $\rho\left(\mathbf{G}_{o p t}^{c}\right), \rho\left(\mathbf{G}_{2}^{c}\right)<\rho\left(\mathbf{G}_{o p t}^{c}\right)$.

\subsection{Adjusted rectangularity feature}

The rectangularity feature scales with the structure size having lower values for small structures. A detector based on such a feature is prone to dismiss small rectangles. On the other hand, false structures of a small size are more frequent. We, therefore, introduce an additional feature $f_{S}$ proportional to the structure size and learn a classifier from the available data in the two-dimensional feature space.

\footnotetext{
${ }^{2}$ Maximal cliques are cliques that are not contained in larger cliques.
}
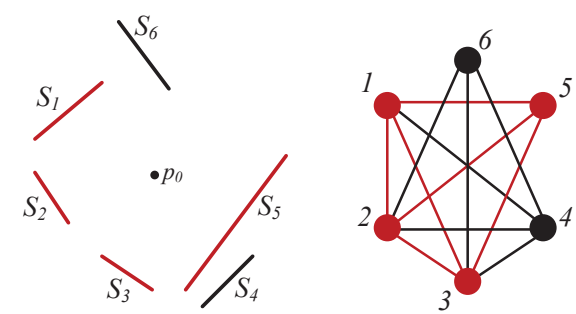

Figure 4. Left: A given set $W=\left\{\mathcal{S}_{1}, \mathcal{S}_{2}, . ., \mathcal{S}_{6}\right\}$ of linear segments around a candidate point $p_{0}$. Right: A graph $\mathbf{G}^{w}$ for the set of linear segments. We assume an angle tolerance $\alpha$ such that all angle constraints are satisfied. Several node pairs of the graph are not connected by an edge due to the convexity constraint, which is not satisfied for an assumed convexity tolerance $t$. The red nodes of the graph are the nodes of the optimal maximal clique $\mathbf{G}_{o p t}^{c}$. The corresponding valid configuration $\mathbf{C}_{o p t}$ is marked in red on the left figure.

This may improve the trade-off between the sensitivity and the number of false detections in comparison to the onedimensional case. We define the size of the structure, represented by the optimal clique $\mathbf{G}_{o p t}^{c} \subseteq \mathbf{G}^{w}$, as

$$
f_{S}\left(\mathbf{G}^{w}\right)=\frac{\sum_{j} l_{j} r_{j}}{\sum_{j} l_{j}}
$$

where the sums are over all nodes of the optimal clique $\mathbf{G}_{o p t}^{c} \cdot f_{S}$ is computed as the weighted distance of the linear segments of $\mathbf{C}_{\text {opt }}$ from the corresponding candidate point, where the weights are segment sizes.

Since only a few positive examples are available in our case, a classification approach should be carefully chosen. The linear classifiers are favorable when there is a danger of overfitting the data due to a limited number of available examples. They also are not computationally demanding. Simple linear classifiers may be powerful enough when used together with a few category-specific features as opposed to the use of many generic features, [34]. We carefully constructed such rectangularity and size features. The normal $w$ of the separating hyperplane of a linear classifier can be found by means of the Fisher Linear Discriminant analysis (FLD). In this approach, the optimal direction is determined such that the data from two classes projected on $w$ is maximally separated. The separation is measured by the squared distance between class means normalized by the sum of their variances $[9,7]$. This approach results in a simple solution represented in terms of class means and covariance matrices. In our case, however, the number of positive examples is very limited and the covariance matrix cannot reliably be estimated.

We optimize the normal direction $w$ based on the large number of available samples from the dominant class of negatives and just a few examples from the class of positives (novelties). Let us define the expected signed distance 
between an arbitrary point $y$ and the distribution $X$ of negatives, both projected to the direction $w$ and normalized by the standard deviation of the projected distribution by

$$
D_{w}(y, X) \equiv \frac{E_{x}\left[w^{T} y-w^{T} x\right]}{\sqrt{E_{x}\left[\left(w^{T} x-w^{T} \mu_{x}\right)^{2}\right]}}=\frac{w^{T}\left(y-\mu_{x}\right)}{\sqrt{w^{T} C_{x} w}},
$$

where $\mu_{x}$ and $C_{x}$ are the mean and the covariance matrix of the distribution $X$, respectively. Next, we define the average signed distance between a set of points $\left\{y_{i}, i=1, \ldots, n\right\}$ and the distribution $X$

$$
\bar{D}_{w}\left(\left\{y_{i}\right\}, X\right) \equiv \frac{1}{n} \sum_{i=1}^{n} D_{w}\left(y_{i}, X\right)=\frac{w^{T}\left(\bar{y}-\mu_{x}\right)}{\sqrt{w^{T} C_{x} w}},
$$

where $\bar{y}=\frac{1}{n} \sum_{i=1}^{n} y_{i}$. We now define the optimal direction $w$ as the direction that maximizes the absolute value of the average signed distance between a set of points corresponding to positive examples and the distribution of the dominant class of negatives $X$, i.e.

$$
w_{\text {opt }} \equiv \underset{w}{\operatorname{argmax}}\left|\bar{D}_{w}\left(\left\{y_{i}\right\}, X\right)\right| .
$$

From Eqs. $(13,14)$ we obtain

$$
w_{\text {opt }}=\underset{w}{\operatorname{argmax}} \frac{\left|w^{T}\left(\bar{y}-\mu_{x}\right)\right|}{\sqrt{w^{T} C_{x} w}} .
$$

It can be shown that

$$
w_{\text {opt }}=C_{x}^{-1}\left(\bar{y}-\mu_{x}\right)
$$

is a solution of Eq. (15). The obtained direction $w_{o p t}$ is similar to the one in the FLD analysis [7]. In contrast to the FLD solution, Eq. (16) includes the covariance matrix of the class of negatives only, preferring the solution in the direction of the small variance of negatives. Negatives are well sampled in our problem and their covariance matrix can be robustly estimated. The positives are treated as deterministic points in the feature space and influence the solution only via their average. Literally, the average only weakly guides the solution showing were the novelties of our interest reside. The samples of $X$ may include outliers. Therefore, in Eq. (16) we use the robust Multivariate Trimming estimates of the mean and the covariance matrix [5].

Given the optimal hyperplane defined by $w_{\text {opt }}$, samples with coordinates $\left(f_{S}, f_{R}\right)$ in feature space can be characterized by the adjusted rectangularity feature

$$
\hat{f}_{R}=\left(f_{S} f_{R}\right) w_{o p t} .
$$

Thereby, the adjusted rectangularity feature is an optimal linear combination of the rectangularity and size features. Note that this approach is not limited to two dimensional feature spaces, but directly extends to higher dimensions.

\section{Experiments}

We evaluate the discrimination ability of the introduced rectangularity features and provide comparison with the NMR measure in [38] and the GODF-based feature in [1] using our implementation, see Sec. 3.3.

\subsection{Data used and preprocessing}

In our experiments we used panchromatic images captured by the GeoEye1 satellite and the red channel of Swiss Topo aerial images. Both types of images are at $0.5 \mathrm{~m}$ resolution. Nine examples of enclosures taken from aerial and satellite images were available for us. A large number of negative examples was generated from $19000 \times 10000$ pixel size satellite image of the Silvretta mountains, which corresponds to about $48 \mathrm{~km}^{2}$. The data stems from a recent archaeological project in the Silvretta mountains [18].

We used the preprocessing flow as in [38]. Bar edges were extracted using the Morphological Feature Contrast based line detector $[40,39]$. This technique extracts linear features, while suppressing texture elements of cluttered background. We also experimented with other approaches $[20,10,28]$, but these are either not sensitive enough to extract faint edges of enclosures, or generate lots of clutter edges depending on the parameters used. The parameterless line segment detector [11], which is known to provide robust results for a large range of images, misses faint edges of enclosures. Extraction of candidate points was carried out by sampling the skeleton points of a complementary binary map of detected bar edges [38]. The second row of Fig. 5 shows examples of maps of bar edges and candidate points for the corresponding images in the first row. Along with the skeleton, we computed the distance transform of the set of bar edges. The values of the distance transform at the candidate points were used to adaptively define the sizes of an analysis window. We discarded all candidate points having a distance smaller than 10 or greater than 90 pixels, which limits the distances between opposite walls of the structures. High contrast texture regions were filtered out using the Morphological Texture Contrast descriptor [40, 39, 37] thresholded with the Otsu method [27]. This filters out urban areas, forests, rocky mountains, and other high contrast texture regions, but preserves individual structures.

\subsection{Measuring discrimination power}

To detect LE, an appropriate threshold on the value of the rectangularity features must be set. Setting a particular threshold defines the true positives rate (TPR) and the false positives rate (FPR), or correspondingly the number of detected true and false positives (TP and FP). In our case, the effectiveness of the rectangularity features is their ability to discriminate LE from irrelevant structures and clutter. A 

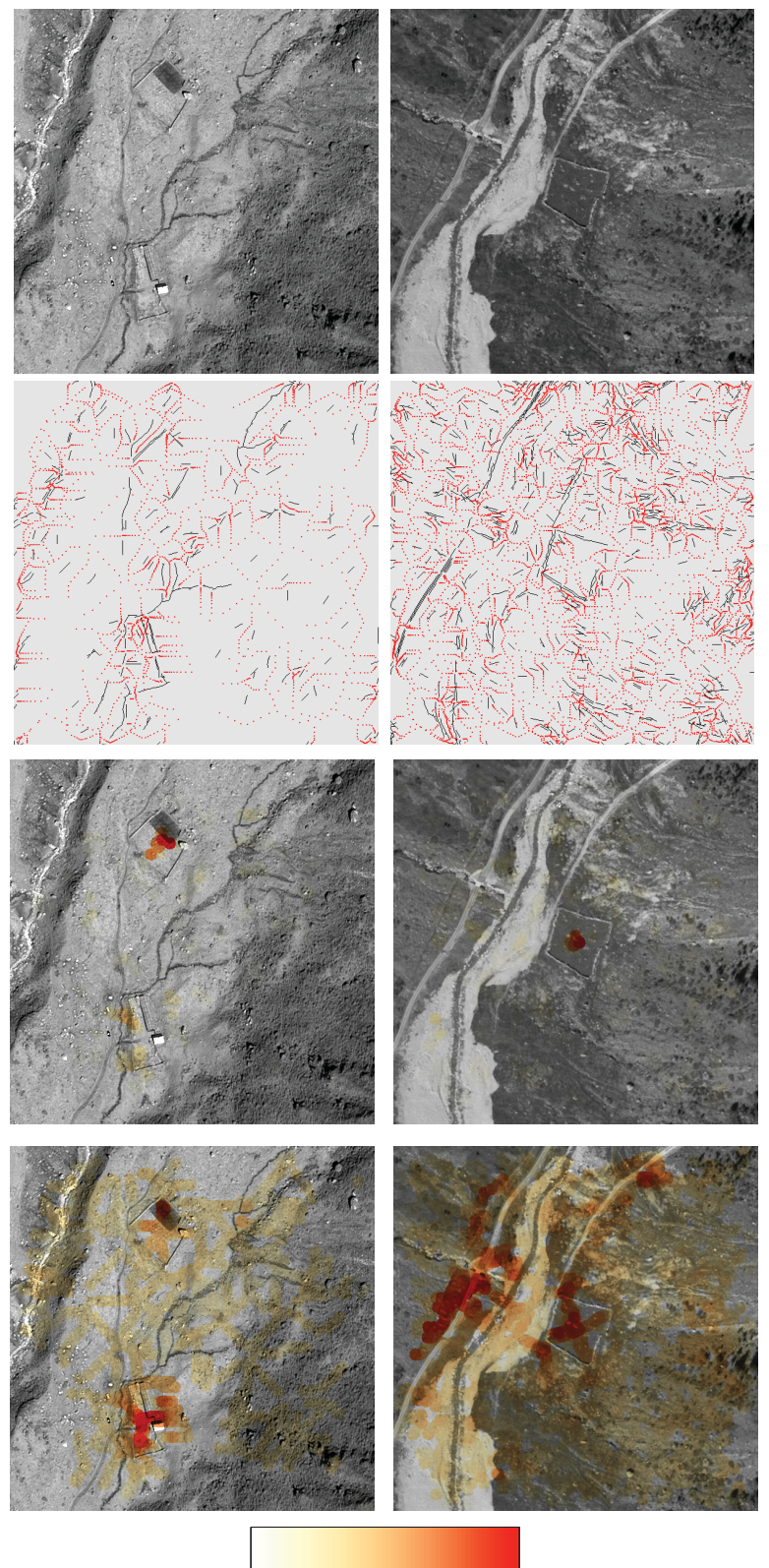

Figure 5. First row: $600 \times 600$ satellite (CGeoEye 2011) and aerial (SWISSTOPO) images of $0.5 \mathrm{~m}$ resolution with structures corresponding to livestock enclosures in Fig. 1. Second row: Bar edges (black) and candidate points (red) generated from the images in the first row. Third row: The rectangularity feature computed at each candidate point and visualized by a colored disk. Fourth row: The GODF-based feature. Color saturation increases and hue is changing from yellow to red for growing values of the features in accordance with the color bar in the bottom.

possible measure of this ability is the minimal number of FP detected with the threshold that insures $T P R \geq \xi$, where $\xi$ is the predefined rate of true positives ${ }^{3}$. We computed $F P$ for $\xi=1$, denoted in the following by $F P_{100}$. This was done by setting the detection threshold to the minimum value of the rectangularity feature computed for nine available positives. Obviously, the threshold used to obtain the detection rate $T P R=1$ on a small number of available examples does not insure a detector with $100 \%$ detection rate. However, it allows us to measure and compare the discrimination ability of the rectangularity features.

We also used an alternative measure of the discrimination ability that is the area under receiver operating characteristic (ROC) curve. It is especially useful in the presence of unbalanced classes $[8,16]$. In contrast to $F P_{100}$, the area under receiver operating characteristic $(A U C)$ does not rely on a particular threshold and a corresponding operating point on the ROC curve, but instead summarizes the detection performance for different values of the threshold.

\subsection{The gradient orientation density function (GODF) based feature}

The GODF-based feature was recently used in [1,23] for detection of buildings. The GODF, denoted $\lambda(\theta)$, captures the distribution of orientations of intensity gradients. The correlation of $\lambda(\theta)$ with a function having two modes separated by $90^{\circ}$ served as a GODF-based feature $f_{G}$ indicating the presence of rectilinear structures. Let $A$ be the neighborhood around a candidate point and let us denote by $g(p)$ the intensity gradient (the Prewitt operator was used) and by $\varphi_{g}(p)$ the gradient orientation at $p . \lambda(\theta)$ is computed as a weighted gradient orientation histogram with gradient magnitudes $\|g(p)\|$ as weights, and discrete orientation $\theta \in[0,180), \theta=k \Delta \theta$, where $k=0,1,2, \ldots$,

$$
\lambda(\theta)=\frac{1}{B} \sum_{p \in A}\|g(p)\| I\left(\theta, \varphi_{g}(p)\right) .
$$

The discrimination step $\Delta \theta$ was set to one. $B$ is a normalizing constant such that $\lambda(\theta)$ is a unit vector ${ }^{4}$, and $I(\theta, \varphi)$ is the indicator function that equals one if $\varphi \in[\theta, \theta+\Delta \theta)$, and zero otherwise. The GODF-based feature $f_{G}$ at the candidate point is then defined as a circular correlation of the orientation histogram $\lambda(\theta)$ with the function $\mathrm{f}_{\Delta 90}$

$$
f_{G}=\max _{\vartheta \in[0,90)} \sum_{\theta} \lambda(\theta) \mathrm{f}_{\Delta 90}((\theta-\vartheta) \text { modulo 180). }
$$

$\mathrm{f}_{\triangle 90}$ is defined in the interval $[0,180)$ and composed of modes separated by $90^{\circ}$. The shape of the modes was the same as for the modes of $\mathrm{f}_{90}$ and $\mathrm{f}_{180}$ in Eq. (7).

\subsection{Results}

The rectangularity feature $f_{R}^{2}$ computed at the candidate points is visualized by colored disks in Fig. 5 (third row).

\footnotetext{
${ }^{3}$ This corresponds to the so-called Neyman-Pearson task [29].

${ }^{4}$ This gave us better results than for $B=\sum_{p \in A}\|g(p)\|$ used in [1].
} 
It was squared in order to visually better distinguish its low and high values. As expected, high values were obtained at positions of LE while zero or low values were obtained at most other candidate positions. Visually similar results are obtained with the NMR measure from [38]. We do not show the corresponding images here (see examples in [38]) due to space limitations. Less convincing results were obtained for the GODF-based feature $f_{G}^{8}$ in Fig. 5 (fourth row). The GODF-based feature was raised to the eighth power, since the second power did not suffice to visually distinguish its low and high values. One can see that the rectangularity feature map is much sparser than the GODF-based feature map. This is partially because the rectangularity feature has zero value for spurious structures with less than three sides, while the GODF-based feature may have only small nonzero values for such structures.

The quantitative measures of performance are summarized in Table 1. The structures were detected out of 403716 candidate positions in the $19000 \times 10000$ pixel image. The results show that the discrimination ability of the adjusted rectangularity feature $\hat{f}_{R}$ is superior to the others. It allows reduction of FP by $24 \%$ relatively to $f_{R}$, which is in turn considerably better than the NMR measure in [38]. Though effective for building detection, the GODF-based feature turned out to be far worse for detecting faint enclosures in cluttered background. This feature is not useful when computed over large windows, where the relative number of points belonging to an enclosure is very low. Fig. 6 shows typical FP obtained for the rectangularity features. These FP were caused by streams and roads. The use of $3 \mathrm{D}$ data (e.g. LiDAR) would allow the discrimination of such FP.
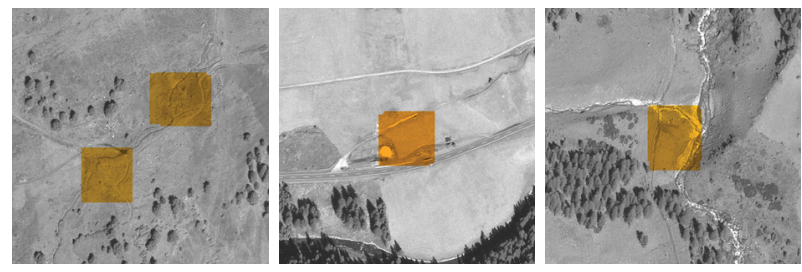

Figure 6. Typical false positives (within color rectangles).

The experiments were carried out using Matlab on a machine with an Intel Core i5 3.3 GHz processor. Generation of candidate locations and computation of the introduced rectangularity features took about two hours. Generation of the NMR measure and the GODF-based feature took about forty and ninety minutes, respectively.

\section{Discussion and Conclusion}

The introduced rectangularity and the adjusted (learnt) rectangularity features have shown good performance discriminating ruined enclosures from irrelevant structures and clutter in remotely sensed images. Due to the inherent diffi-

\begin{tabular}{r|llll} 
& $f_{G}$ & $f_{N}$ & $f_{R}$ & $\hat{f}_{R}$ \\
\hline$F P_{100}$ & 6522 & 334 & 133 & 101 \\
$A U C \times 10^{2}$ & 99.683 & 98.292 & 99.993 & 99.994 \\
\hline
\end{tabular}

Table 1. Detection performance using the GODF-based feature $f_{G}$ ([1]), the normalized maximum rectangularity measure $f_{N}$ ([38]), the rectangularity $f_{R}$, and the adjusted rectangularity $\hat{f}_{R}$ features.

culties of our problem, such a performance is hardly achievable with other approaches for detection of rectangular contours, nor with related approaches, e.g. for detection of buildings. As an example we have shown that the GODFbased feature used for detection of buildings (e.g. in [1]) reveals a poor discrimination ability for our task. Note that we did not compare the rectangularity features with the whole approach developed in [1], because it is based on additional features not appropriate in the case of enclosures. Also, parameters of the data model would be hard to estimate from only few available examples of enclosures. We have also tested other methods for building detection (e.g. [31, 12]) applied to detection of livestock enclosures. Unfortunately, these methods completely fail to detect the enclosures, preventing us from reporting the corresponding quantitative comparison.

In general, methods for building detection are not suitable for our case because of considerably lower heights (related to low feature contrasts) and feature sizes (ruined walls versus building rooftops), and due to the absence of various cues (roof colors, roof homogeneity, shadows, 3D cues, etc.). Some walls or parts of them may be missing or may also be missed in the edge extraction (the width of linear features does not exceed two pixels in images of $0.5 \mathrm{~m}$ resolution). Various irrelevant structures (trails, streams, rocks etc.) with sizes or/and reflectance properties similar to those of enclosure walls may occasionally form rectilinear configurations. In contrast to enclosures, building rooftops are much more distinctive structures.

It is interesting to investigate the usefulness of the rectangularity features for detection of targets other than livestock enclosures. We believe, for example, that our approach may have comparative performance for detection of abandoned buildings or other architectural structures in rural or mountainous areas ${ }^{5}$. Building contours can be extracted with standard edge detection algorithms and used as an input for our approach. Note that, while building detection problem can be transformed to detection of enclosures ${ }^{6}$, it does not work in the opposite direction. We also plan to incorporate additional features and apply the FLD-based detector to vast alpine areas in order to spot unknown livestock enclosures.

\footnotetext{
${ }^{5}$ In urban areas our approach is likely to be too sensitive. Walls of adjacent buildings may cause a large number of false detections within urban areas.

${ }^{6}$ Cues such as roof color or corner features will not be used, however.
} 


\section{References}

[1] C. Benedek, X. Descombes, and J. Zerubia. Building development monitoring in multitemporal remotely sensed image pairs with stochastic birth-death dynamics. IEEE Trans. Pattern Anal. Mach. Intell., 34(1):33-50, 2012.

[2] C. Bron and J. Kerbosch. Algorithm 457: finding all cliques of an undirected graph. Commun. ACM, 16(9):575-577, Sept. 1973.

[3] A. Croitoru and Y. Doytsher. Right-angle rooftop polygon extraction in regularised urban areas: Cutting the corners. The Photogrammetric Record, 19(108):311-341, 2004.

[4] X. Descombes and J. Zerubia. Marked point process in image analysis. Signal Processing Magazine, IEEE, 19(5):7784, 2002

[5] S. J. Devlin, R. Gnanadesikan, and J. R. Kettenring. Robust estimation of dispersion matrices and principal components. Journal of the American Statistical Association, 76(374):354-362, 1981

[6] R. O. Duda and P. E. Hart. Use of the Hough transformation to detect lines and curves in pictures. Commun. ACM, 15(1):11-15, Jan. 1972.

[7] R. O. Duda and P. E. Hart. Pattern Classification and Scene Analysis. John Willey \& Sons, 1973.

[8] T. Fawcett. An introduction to ROC analysis. Pattern Recogn. Lett., 27(8):861-874, June 2006.

[9] K. Fukunaga. Introduction to Statistical Pattern Recognition. Academic Press., 1990.

[10] C. Grigorescu, N. Petkov, and M. Westenberg. Contour and boundary detection improved by surround suppression of texture edges. Image and Vision Computing, 22:609 $622,2004$.

[11] R. Grompone von Gioi, J. Jakubowicz, J.-M. Morel, and G. Randall. LSD: A fast line segment detector with a false detection control. IEEE Trans. Pattern Anal. Mach. Intell. 32(4):722-732, Apr. 2010.

[12] C. R. Jung and R. Schramm. Rectangle detection based on a windowed Hough transform. In Proceedings of the Computer Graphics and Image Processing (SIBGRAPI), XVII Brazilian Symposium, pages 113-120, 2004.

[13] C. G. Keller, C. Sprunk, C. Bahlmann, J. Giebel, and G. Baratoff. Real-time recognition of US speed signs. In Intelligent Vehicles Symposium, pages 518-523. IEEE, 2008.

[14] T. Kim and J.-P. Muller. Development of a graph-based approach for building detection. Image Vision Comput., 17(1):3-14, 1999.

[15] S. Krishnamachari and R. Chellappa. Delineating buildings by grouping lines with MRFs. IEEE Transactions on Image Processing, 5(1):164-168, 1996.

[16] W. J. Krzanowski and D. J. Hand. ROC Curves for Continuous Data. Chapman \& Hall/CRC, 2009.

[17] L. Lam, S.-W. Lee, and C. Y. Suen. Thinning methodologiesa comprehensive survey. IEEE Transactions on Pattern Analysis and Machine Intelligence, 14(9):869 - 885, 1992. p.879.

[18] K. Lambers and I. Zingman. Towards detection of archaeological objects in high-resolution remotely sensed images: the Silvretta case study. In G. Earl et al., editors, Archaeology in the digital era (e-papers). Proc. of Computer Applications and Quantitative Methods in Archaeology, volume II, pages 781-791, Southampton, UK, March 2012. Amsterdam University Press.

[19] C. Lin and R. Nevatia. Building detection and description from a single intensity image. Computer Vision and Image Understanding, 72(2):101-121, 1998.

[20] T. Lindeberg. Edge detection and ridge detection with automatic scale selection. International Journal of Computer Vision, 30(2):117-156, 1998.

[21] Y. Liu, T. Ikenaga, and S. Goto. An MRF model-based approach to the detection of rectangular shape objects in color images. Signal Processing, 87(11):2649-2658, 2007.

[22] G. B. Loy and N. M. Barnes. Fast shape-based road sign detection for a driver assistance system. In 2004 IEEE/RSJ International Conference on Intelligent Robots and Systems, Sendai, Japan, pages 70-75, 2004.

[23] A. Manno-Kovacs and T. Sziranyi. Multidirectional Building Detection in Aerial Images Without Shape Templates. ISPRS - International Archives of the Photogrammetry, Remote Sensing and Spatial Information Sciences, (1):010000-232, May 2013.

[24] H. Mayer. Automatic object extraction from aerial imagery a survey focusing on buildings. Computer Vision and Image Understanding, 74(2):138-149, 1999.

[25] H. Moon, R. Chellappa, and A. Rosenfeld. Optimal edgebased shape detection. IEEE Transactions on Image Processing, 11(11):1209-1227, 2002.

[26] M. Ortner, X. Descombes, and J. Zerubia. A marked point process of rectangles and segments for automatic analysis of digital elevation models. Pattern Analysis and Machine Intelligence, IEEE Transactions on, 30(1):105-119, 2008.

[27] N. Otsu. A Threshold Selection Method from Gray-level Histograms. IEEE Transactions on Systems, Man and Cybernetics, 9:62-66, Jan. 1979.

[28] G. Papari and N. Petkov. An improved model for surround suppression by steerable filters and multilevel inhibition with application to contour detection. Pattern Recognition, 44:1999 - 2007, 2011.

[29] M. I. Schlesinger and V. Hlavac. Ten lectures on statistical and structural pattern recognition. Springer, 2002.

[30] B. Sirmacek and C. Unsalan. Urban-area and building detection using SIFT keypoints and graph theory. IEEE T. Geoscience and Remote Sensing, 47(4):1156-1167, 2009.

[31] B. Sirmacek and C. Unsalan. A probabilistic framework to detect buildings in aerial and satellite images. IEEE Tran. Geoscience and Remote Sensing, 49(1-1):211-221, 2011.

[32] Ø. D. Trier, S. Ø. Larsen, and R. Solberg. Automatic detection of circular structures in high-resolution satellite images of agricultural land. Archaeological Prospection, 16:1-15, 2009.

[33] Y. Verdie and F. Lafarge. Detecting parametric objects in large scenes by Monte Carlo sampling. International Journal of Computer Vision, 106(1):57-75, 2014.

[34] M. Vidal-Naquet and S. Ullman. Object recognition with informative features and linear classification. In Proc. of the 
International Conference on Computer Vision, pages 281288 vol.1, Oct 2003.

[35] Z. Yu and C. Bajaj. Detecting circular and rectangular particles based on geometric feature detection in electron micrographs. Journal of Structural Biology, 145(12):168 - 180, 2004.

[36] Y. Zhu, B. Carragher, F. Mouche, and C. S. Potter. Automatic particle detection through efficient hough transforms. IEEE Trans. Med. Imaging, 22(9):1053-1062, 2003.

[37] I. Zingman, D. Saupe, and K. Lambers. Morphological operators for segmentation of high contrast textured regions in remotely sensed imagery. In Proc. of the IEEE Int. Geoscience and Remote Sensing Symposium, pages 3451-3454, Munich, Germany, July 2012.

[38] I. Zingman, D. Saupe, and K. Lambers. Automated search for livestock enclosures of rectangular shape in remotely sensed imagery. In L. Bruzzone, editor, Proc. SPIE, Image and Signal Processing for Remote Sensing XIX, volume 8892, pages 88920F-1 - 88920F-11, Dresden, Germany, 2013.

[39] I. Zingman, D. Saupe, and K. Lambers. Detection of texture and isolated features using alternating morphological filters. In C. Hendriks, G. Borgefors, and R. Strand, editors, Mathematical Morphology and Its Applications to Signal and Image Processing, volume 7883 of Lecture Notes in Computer Science, pages 440-451. Springer, 2013.

[40] I. Zingman, D. Saupe, and K. Lambers. A morphological approach for distinguishing texture and individual features in images. Pattern Recognition Letters, 47:129 - 138, 2014. Advances in Mathematical Morphology. 